\title{
Multiplexed photo-activation of mRNA with single-cell resolution
}

\author{
Dongyang Zhang $\$$, Shuaijiang Jin $\$$, Xijun Piao, and Neal K. Devaraj*
}

\section{ABSTRACT}

We demonstrate sequential optical activation of two types of mRNAs in the same mammalian cell through the sequential photocleavage of small molecule caging groups ('photo-cages') tethered to the 5' untranslated region (5'-UTR) of an mRNA. Synthetic 'photo-cages' were conjugated onto target mRNA using RNA-TAG, an enzymatic site-specific RNA modification technique. Translation of mRNA was severely reduced upon conjugation of the 'photo-cages' onto the 5'UTR. However, subsequent photo-release of the 'cages' from the mRNA transcript triggered activation of translation with single-cell spatiotemporal resolution. To achieve sequential photoactivation of two mRNAs in the same cell, we synthesized a pair of 'photo-cages' which can be selectively cleaved from mRNA upon photo-irradiation with different wavelengths of light. Sequential photo-activation of two mRNAs enabled precise optical control of translation of two unique transcripts. We believe that this modular approach to precisely and rapidly control gene expression will serve as a powerful tool in future biological studies that require controlling translation of multiple transcripts with high spatiotemporal resolution.

\section{INTRODUCTION}

The ability to precisely control gene expression is important for a wide range of applications in basic biological research, genetics, as well as gene therapies. [1-2] Stimuliresponsive control in confined time and space is enabled by inducible gene expression systems. For instance, small-molecule inducers, such as doxycycline, have been widely used in regulating synthetic gene circuits. [3] However, chemical inducers have slow diffusion rates, potential toxicity, and off-target effects on living systems, limiting their utility when high spatiotemporal resolution is required. [4] In contrast, optogenetic approaches utilize light as an external stimulus to regulate cellular processes. [5] Light irradiation is convenient to apply to biological samples such as live cells and organisms. The adverse effects of light-irradiation on living systems can be minimized by optimizing the wavelength and irradiation period of the light source. Perhaps most importantly, light can be applied with high spatial-temporal resolution, offering fast activation and deactivation dynamics. [6-7]

There has been an extensive body of research aimed at developing optogenetic tools to regulate gene expression. In a common strategy, a light-sensitive protecting group ('photo-cage') is chemically installed on a biologically relevant target (e.g. metabolites, oligonucleotides, or proteins) rendering the substrate inactive. Subsequently, light irradiation triggers the release of the 'photo-cage' from the target biological molecule, restoring its cellular activity. [8-10] For instance, optogenetic approaches often involve the installation of light-responsive protein domains or amino acids to achieve photo-chemical manipulation of proteins such as nucleases, proteases and 
transcription factors. [11-16] Caged oligonucleotides have also been used to control gene expression at the level of transcription or translation. [17-24] By conjugating 'photo-cages' onto nucleotide bases, hybridization between the antisense oligonucleotide and the target DNA/RNA can be controlled precisely using light. While most studies have focused on optogenetic control of transcription, there are advantages to methods that optically control gene expression at the level of translation. [25-29, 41] Since mRNA can be processed by cellular translation machinery immediately upon cytoplasm entry, controlling gene expression through direct manipulation of mRNA provides more rapid changes in cellular protein concentration compared to the regulation of transcription. [30] Moreover, in-vitro transcribed mRNA (IVT-mRNA) is only transiently active in cytosol and is completely degraded via cellular metabolism, typically within 24 hours. Thus, unlike the use of plasmid DNA or viral vectors which may be integrated into the cellular genome, mRNA-based gene expression regulation systems do not pose the risk of insertional mutagenesis. [31] Thus, a technique that enables optogenetic manipulation of translation would benefit from the high spatial-temporal resolution inherent to optical control as well as fast dynamics of mRNA processing.

Previously, we demonstrated a technique which enabled precise optical control of mRNA translation through the conjugation of light-sensitive 'cages' onto IVT-mRNA. [32] Laser irradiation $(405 \mathrm{~nm})$ on live cells removed the 'photo-cages' from cytoplasmic mRNA, subsequently activating translation with single cell precision. We speculated we could significantly expand this tool by enabling sequential photo-activation of two mRNAs within the same cell. Here, leveraging the multiplex capability of our mRNA caging/uncaging platform, we describe a sequentially light-activated translation regulatory system that utilizes two 'photo-cages' to cage two types of mRNAs. Irradiation with longer wavelength light (456-488 nm) activates one mRNA, while subsequent irradiation with shorter wavelength light (365-405 nm) activates the other mRNA. This multiplexed gene expression regulatory system provides a high degree of flexibility and shows potential for enabling the study of multiple regulatory genes with high spatial-temporal resolution.

\section{RESULTS AND DISCUSSION}

To allow for site-specific and covalent conjugation of small molecule effectors onto target mRNA, we previously developed a technology named RNA transglycosylation at guanosine (RNA-TAG), which utilizes a bacterial tRNA guanine transglycosylase (TGT) to exchange a guanine nucleobase within a specific 17-nucleotide RNA stem-loop structure ('Tag') with synthetic enzyme substrate analogs (Figure S1, Figure 1A). [33-34] In E. Coli, tRNAs carrying Asn, Asp, His and Tyr are post-transcriptionally modified by TGT, which exchanges the guanine at the wobble position of the anticodon loop of the tRNA with the enzyme's natural substrate pre-

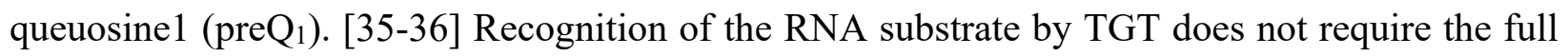
sequence of tRNA. Instead, a minimal 17-nucleotide RNA stem-loop from the anticodon loop of 
the tRNA (the 17-nucleotide 'Tag' sequence) is sufficient to promote TGT recognition and labeling. [37-38] TGT also accepts a wide range of synthetic $\mathrm{preQ}_{1}$ derivatives as small molecule substrates. By genetically inserting the 'Tag' sequence into an RNA of interest, we previously demonstrated TGT labeling is able to covalently conjugate a variety of functional small molecules, such as fluorophores and affinity tags, site-specifically onto the target RNA. [33-34] The versatility of this RNA modifying platform enabled us to adapt this technique to regulate translation, through conjugation of small-molecule effectors directly onto an mRNA transcript.

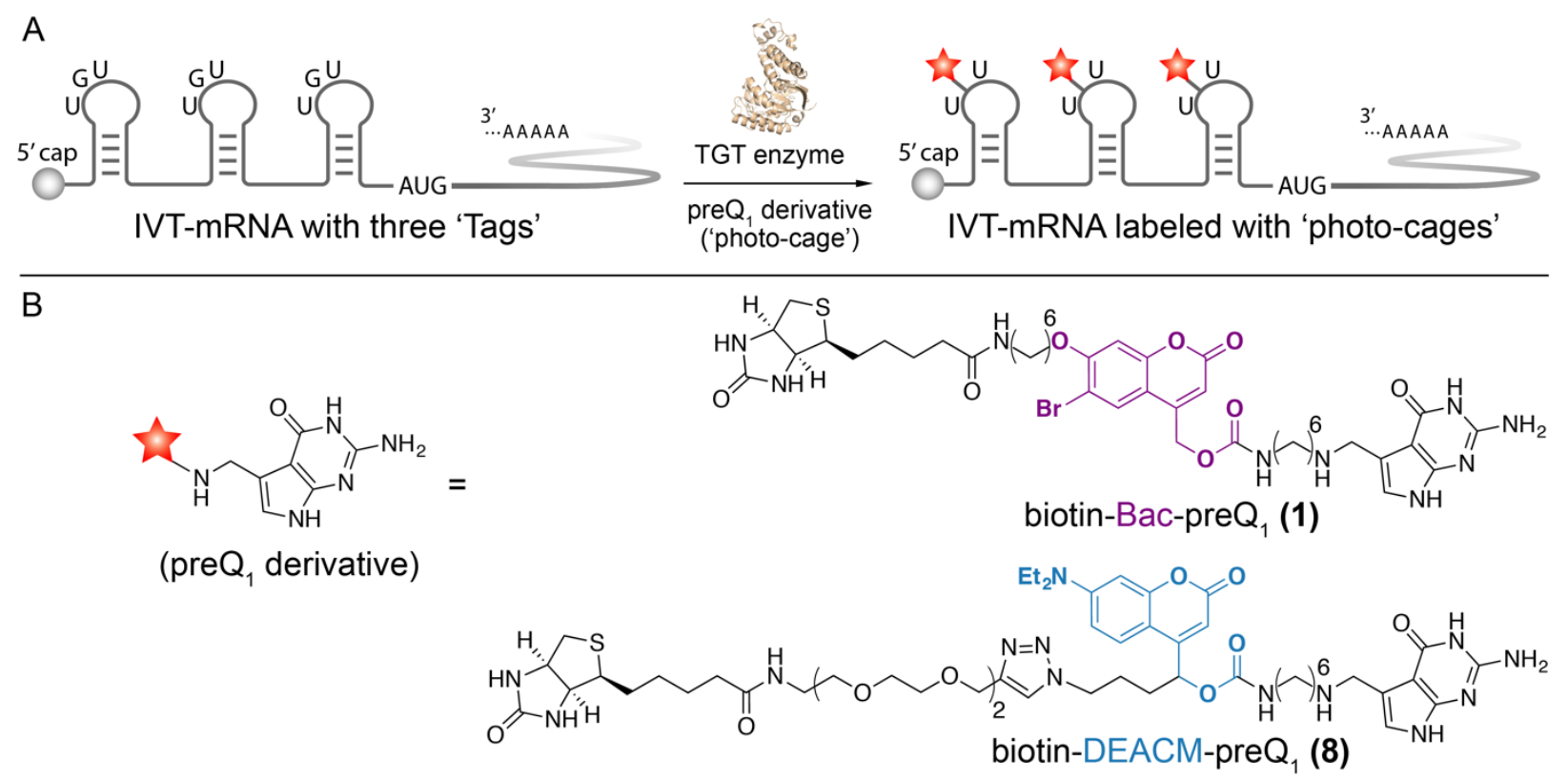

Figure 1. mRNA photo-caging using the RNA-TAG technique. A) To facilitate TGT enzymatic labeling, three enzyme recognition sequences, 'Tags', are genetically inserted along the 5'-UTR of an IVT-mRNA. Subsequent conjugation of the 'photo-cages' severely reduces mRNA translation activity. B) Chemical structures of two sequentially activable preQ ${ }_{1}$ derivatives ('photo-cages'), biotin-Bac-preQ ${ }_{1}$ and biotin-DEACM-preQ 1 .

To achieve optical-control of mRNA translation, we covalently conjugated the synthetic 'photo-cage', biotin-Bac-preQ ${ }_{1}$ (1), at three different locations along the 5'-UTR of a mature IVTmRNA (Figure 1). The first conjugation site was located adjacent to the 5'-cap of the mRNA, specifically, at the 11 th base after the 5 '-cap. The second conjugation site was located in the middle of the 5'-UTR. The third conjugation was located at the 11th base upstream of the AUG start codon (Figure 1A). To determine the effect of such conjugation on translation, both the labeled and unlabeled IVT-mRNAs were delivered into mammalian cells through transient transfection. As a result, translation efficiency of the labeled IVT-mRNA was severely reduced to approximately $10 \%$ of the activity relative to the unlabeled IVT-mRNA. [32] We hypothesized that this phenomenon is due to the steric hindrance of these bulky 'photo-cages' conjugated at the 5'-UTR, which is where translation initiation takes place. Upon irradiation with $405 \mathrm{~nm}$ laser light, the Bac linker 
was photo-cleaved, leaving a minimal amine residue on the mRNA. As a result, translation activity of the IVT-mRNA was recovered after photo-uncaging, demonstrating successful photo-activation of gene expression. Importantly, mRNA translation was only observed in laser irradiated cells, and not in adjacent cells, demonstrating single-cell resolution of activation.
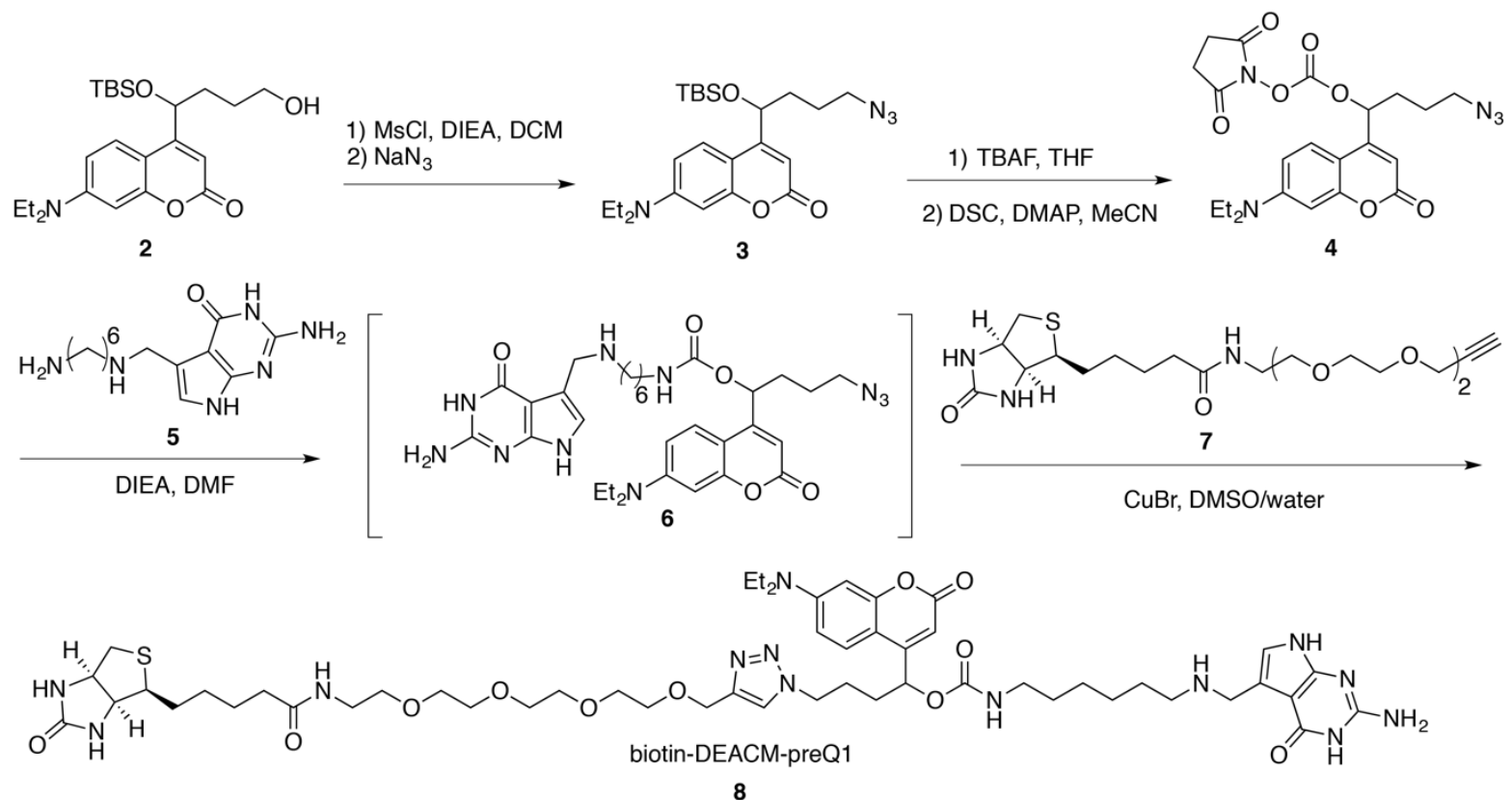

Scheme 1. Synthesis of the preQ1-DEACM-biotin (8). Boc = tert-butyloxycarbonyl protecting group, $\mathrm{MsCl}=$ methanesulfonyl chloride, $\mathrm{DCM}=$ dichloromethane, $\mathrm{TBS}=$ tert-butyldimethylsilyl protective group, TBAF $=$ tetra- $n$-butylammonium fluoride, THF $=$ tetrahydrofuran, DSC $=\mathrm{N}-$ succinimidyl carbonate, DMAP = 4-dimethylaminopyridine, DIEA = N,N-diisopropylethylamine, $\mathrm{DMF}=$ dimethylformamide, $\mathrm{DMSO}=$ dimethyl sulfoxide.

Previously, we chose the photo-cleavable linker 6-bromo-7-aminoethoxycoumarin-4ylmethoxycarbonyl (Bac) to synthesize the 'photo-cage', biotin-Bac-preQ 1 (1) (Figure 1B). [32] To allow for sequential photo-activation of mRNA translation, an additional photo-sensitive linker that is responsive to a longer wavelength of light was desired. Inspired by previously reported work, [39] we chose to explore [7-(diethylamino)coumarin-4-yl]-methyl (DEACM) as an additional photo-activable linker that can be sequentially activated. The DEACM linker has a wide absorbance spectrum and was previously reported to be cleaved in cellular conditions by irradiation with $470 \mathrm{~nm}$ light. [40] Thus, the DEACM linker should form a sequentially photoactivable linker pair with our previously reported Bac linker, which is uncaged by irradiation with 365-405 nm light. To synthesize the new 'photo-cage' biotin-DEACM-preQ 1 (8) (Scheme 1), the DEACM-based building block (2) was subjected to sequential mesylation and azidation to generate azide compound (3). Next, the DEACM NHS-ester (4) was obtained through deprotection of the silica protecting group and N-succinimidyl carbonate (DSC) treatment. Subsequently, the 
preQ $_{1}$ derivative (5) was coupled to the DEACM NHS-ester (4) to yield the preQ ${ }_{1}-D_{E A C M}$ conjugate (6). To introduce a biotin affinity handle to facilitate purification of the labeled mRNAs, the preQ 1 -DEACM conjugate (6) was further coupled with commercially available biotin-PEG 4 alkyne (7) via click chemistry to obtain the final TGT enzymatic substrate biotin-DEACM-preQ ${ }_{1}$ (8).

A

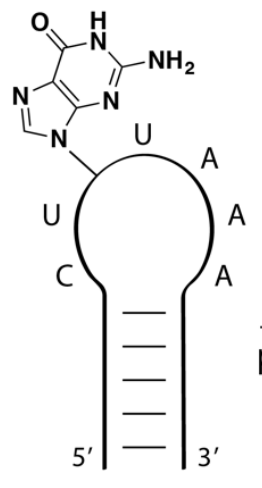

RNA 'Tag' oligo

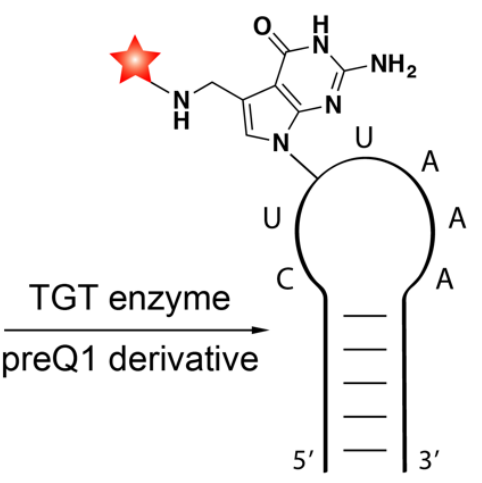

Labeled 'Tag' oligo
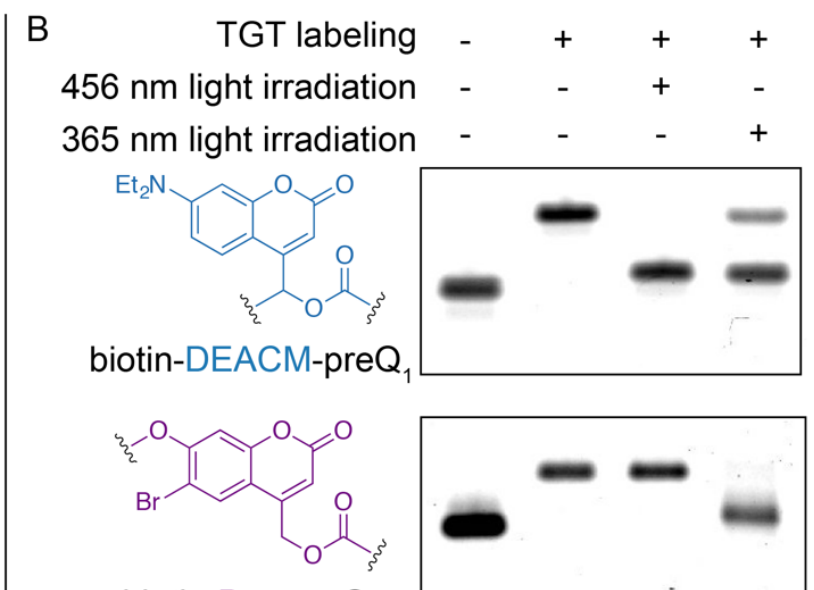

biotin-Bac-preQ 1

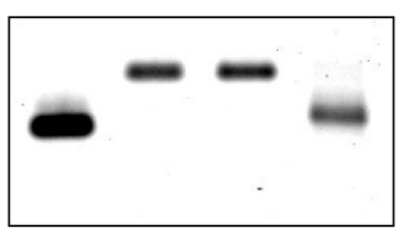

Figure 2. TGT labeling of the RNA 'Tag' oligo and in-vitro sequential photo-uncaging. A) Invitro conjugation of RNA 'Tag' oligo with preQ ${ }_{1}$ derivatives through RNA-TAG. B) Denaturing $18 \%$ PAGE analysis of enzymatic labeling and subsequent photo-uncaging of the RNA 'Tag' oligos. Compared to the unlabeled RNA oligo, an upper gel shift in the second column demonstrates covalent attachment of the corresponding 'photo-cage' onto the RNA oligo. Lower gel shift in the third or fourth column demonstrates photo-release of the 'photo-cage' from the RNA oligo.

To demonstrate that biotin-Bac-preQ1 (1) and biotin-DEACM-preQ $Q_{1}$ (8) can be sequentially released from RNA upon photo-irradiation with two wavelengths of light, in-vitro uncaging of labeled RNA oligos was performed using either $365 \mathrm{~nm}$ or $456 \mathrm{~nm}$ light followed by denaturing polyacrylamide gel electrophoresis (denaturing-PAGE) analysis. The 'Tag' oligo was used as RNA substrate for TGT labeling (Figure 2A). To covalently conjugate the photo-cage onto the 'Tag' oligo, TGT labeling using either biotin-Bac-preQ ${ }_{1}$ or biotin-DEACM-preQ ${ }_{1}$ as small molecule substrate was carried out in a dark room with minimal red ambient light to prevent undesired degradation of the light-sensitive $\mathrm{preQ}_{1}$ derivatives. Labeled 'Tag' oligo was further purified by streptavidin-biotin pull-down followed by denaturing-PAGE analysis. Covalent conjugation of the 'photo-cage' increased the molecular weight of the 'Tag' oligo, resulting in significant RNA band shifts shown in denaturing-PAGE (Figure 2B). [32-33] To trigger the cleavage of the 'photo-cage', the 'Tag' oligo labeled with biotin-DEACM-preQ 1 was irradiated with a $456 \mathrm{~nm}$ lamp, while the 'Tag' oligo labeled with biotin-Bac-preQ 1 was irradiated with a $365 \mathrm{~nm}$ lamp. We observed that upon irradiation with $456 \mathrm{~nm}$ light, only biotin-DEACM-preQ ${ }_{1}$ 
was released from the 'Tag' oligo, not the biotin-Bac-preQ 1 . Biotin-Bac-preQ ${ }_{1}$ was released from the 'Tag' oligo upon irradiation with $365 \mathrm{~nm}$ light. Thus, by using $456 \mathrm{~nm}$ and $365 \mathrm{~nm}$ light sources, sequential release of the 'photo-cages' from RNA was achieved.
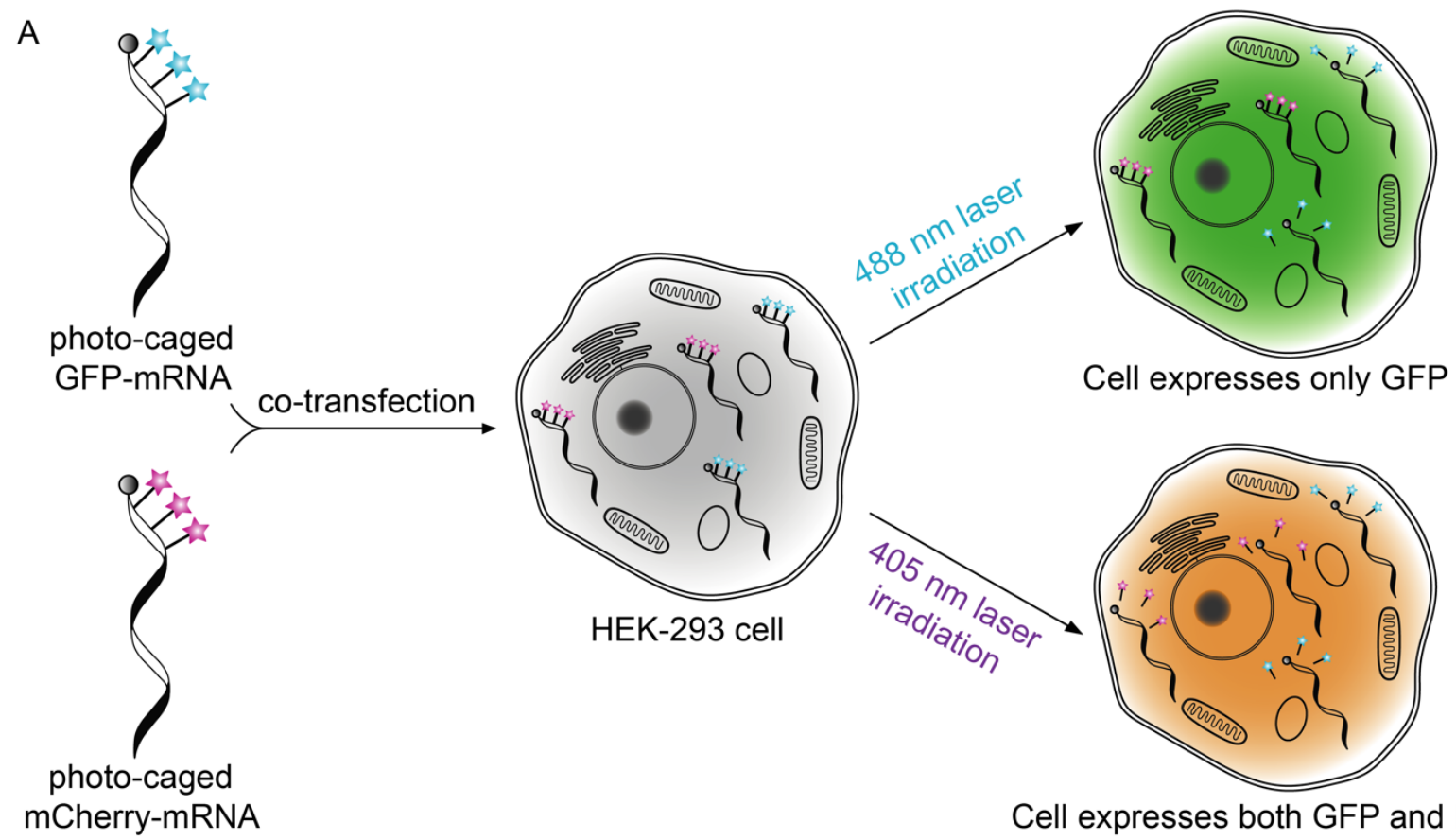

mCherry-mRNA

Cell expresses both GFP and RFP

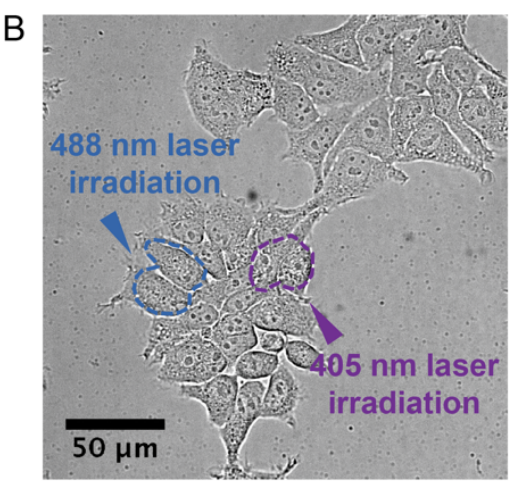

Bright field

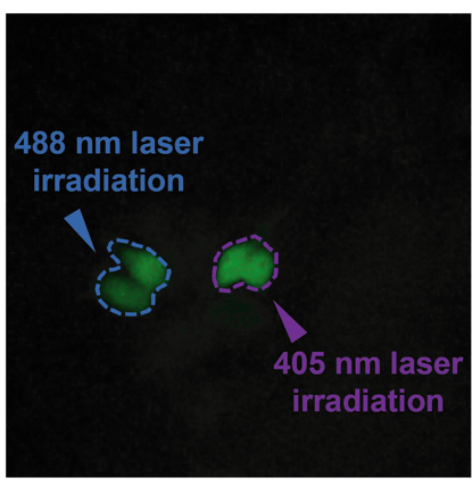

GFP channel

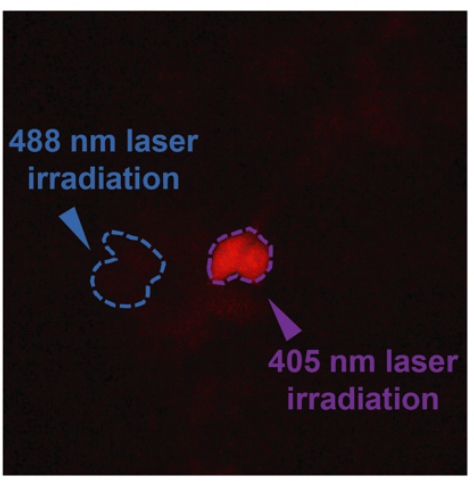

RFP channel

Figure 3. Live cell photo-activation of mRNA translation. A) HEK-293 cells are co-transfected with caged GFP-mRNA and caged mCherry-mRNA, followed by photo-uncaging with either 488 $\mathrm{nm}$ or $405 \mathrm{~nm}$ laser irradiation. B) Live cell fluorescence imaging. Selected cells (circled in blue) irradiated with $488 \mathrm{~nm}$ laser only express GFP, shown as green cells in the GFP channel and dark cells in the RFP channel. Selected cells (circled in purple) irradiated with $405 \mathrm{~nm}$ light express both GFP and RFP. Scale bar $=50 \mu \mathrm{m}$.

Having demonstrated that biotin-DEACM-preQ ${ }_{1}$ and biotin-Bac-preQ ${ }_{1}$ can be sequentially released from RNA oligos in-vitro, we examined whether these 'photo-cages' can be sequentially photo-released from mRNA in live mammalian cells. Mature IVT-mRNAs coding for either the 
green fluorescent protein (GFP) or the red fluorescent protein (RFP), with three TGT labeling sites located along the 5'-UTR, were synthesized following a previously reported mRNA transcription and maturation protocol. [32] Using TGT enzymatic labeling, the mature IVT-mRNA coding for GFP was conjugated with biotin-DEACM-preQ ${ }_{1}$ while the IVT-mRNA coding for RFP was conjugated with biotin-Bac-preQ ${ }_{1}$. To get rid of unlabeled IVT-mRNAs, biotin-streptavidin affinity purification was performed (60\% recovery). Equal amounts of photocaged IVT-mRNAs coding for GFP or RFP were co-transfected into HEK-293 cells using lipofectamine reagent (Figure 3A). mRNA translation activity was quantified by fluorescence imaging. Minimal translation activity was observed for the photocaged mRNAs, shown as dark cells (Figure 3B). Two hours post transfection, selected cells were irradiated with either $488 \mathrm{~nm}$ or $405 \mathrm{~nm}$ wavelength of laser light to trigger the release of the 'photo-cages' from mRNA. To allow time for sufficient mRNA translation and maturation, cells were imaged 8 hours after laser irradiation. As expected from our in-vitro studies, the $488 \mathrm{~nm}$ irradiation only triggered the release of the biotin-DEACM-preQ ${ }_{1}$ from mRNA. As a result, recovered protein expression of GFP was observed only in cells that were irradiated with the $488 \mathrm{~nm}$ laser (Figure 3B). Importantly, expression of RFP was not observed in these cells, which was expected because the biotin-BacpreQ $_{1}$ used to cage the RFP-mRNA is not responsive to $488 \mathrm{~nm}$ irradiation. In contrast, expression of both GFP and RFP was observed in cells that were irradiated with $405 \mathrm{~nm}$ wavelength of laser, because both biotin-Bac-preQ 1 and biotin-DEACM-preQ 1 have absorbance at $405 \mathrm{~nm}$ wavelength. These observations were consistent with our in-vitro uncaging experiments, that the longer wavelength of light only triggered the release of biotin-DEACM-preQ 1 , whereas the shorter wavelength of light triggered the release of both 'photocages' from mRNA. Moreover, expression of fluorescent protein was only observed in laser irradiated cells, not in adjacent cells, demonstrating photo-activation of gene expression with high cellular resolution.

Next, we demonstrated sequential photo-activation of two mRNAs in the same living cell. Caged IVT-mRNAs coding for GFP and RFP were co-transfected into HEK-293 cells. Two hours post transfection, the selected cell was first irradiated with $488 \mathrm{~nm}$ laser light to trigger the release of biotin-DEACM-preQ ${ }_{1}$ from GFP-mRNA. Five hours post transfection, the same cell was then irradiated with $405 \mathrm{~nm}$ laser light to trigger the release of the biotin-Bac-preQ 1 from RFP-mRNA. Cells were continuously imaged to quantify protein expression level (Figure 4A). As shown in the fluorescence images, the first irradiation with $488 \mathrm{~nm}$ laser light triggered the expression of GFP, while the expression of RFP remained silenced. As expected, the subsequent irradiation with 405 $\mathrm{nm}$ laser light activated the expression of RFP. GFP and RFP expression levels in laser irradiated cells were quantified by measuring fluorescence intensity (Figure 4B). Using this model, we demonstrated sequential photo-activation of two mRNAs in the same cell by using two wavelengths of lights. 

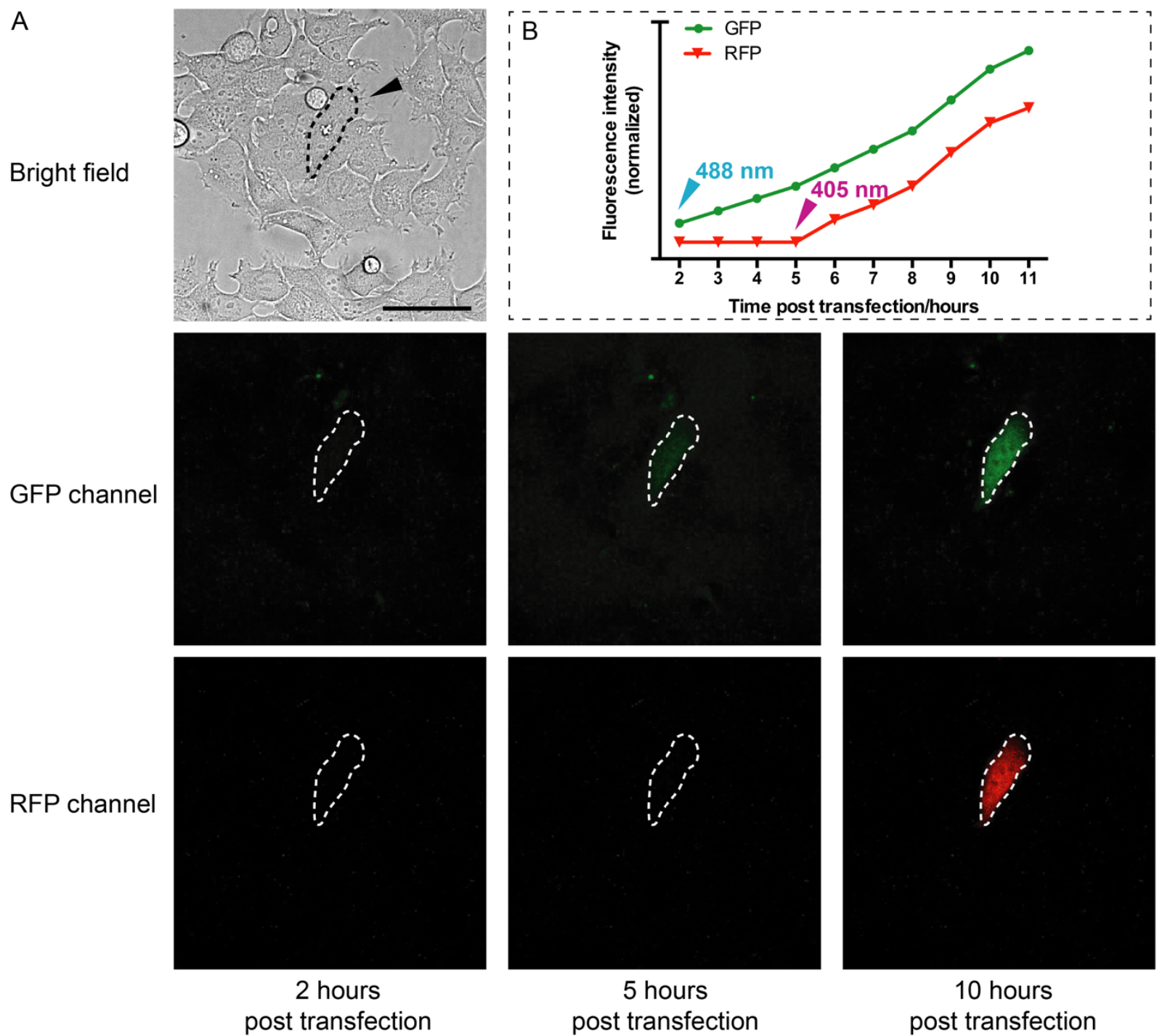

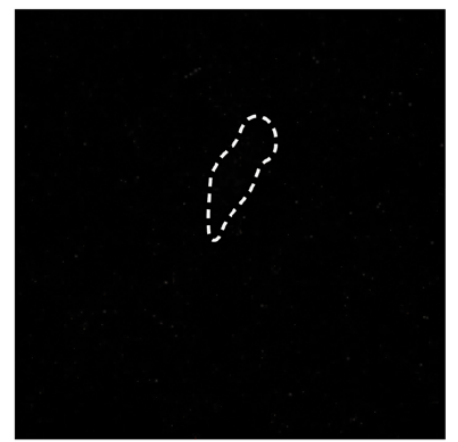

5 hours

post transfection

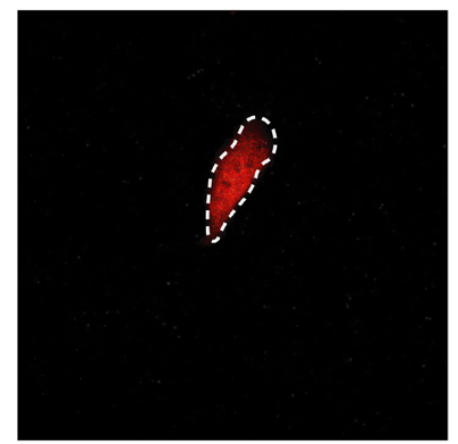

10 hours

post transfection

Figure 4. Live cell sequential photo-activation of two mRNAs within the same cell. A) A selected cell (circled in dash line) was first irradiated with $488 \mathrm{~nm}$ laser light to activate GFPmRNA. Three hours after the first photo-uncaging event, the same cell was irradiated with $405 \mathrm{~nm}$ laser light to activate RFP-mRNA (Scale bar $=50 \mu \mathrm{m})$. B) Normalized average fluorescence intensity of GFP and RFP from this selected cell is plotted against time (hours post transfection).

\section{CONCLUSION}

In conclusion, we have developed a technique that allows sequential photo-activation of two mRNAs with single-cell resolution. We demonstrated the synthesis and photochemical properties of two 'photo-cages', biotin-Bac-preQ ${ }_{1}$ (1) and biotin-DEACM-preQ 1 (8). These 'photocages' were covalently and site-specifically conjugated onto the 5'-UTR of mRNA through TGT enzymatic labeling. As a result, translation efficiency of the labeled mRNA was severely diminished compared to the unlabeled mRNA. This pair of 'photo-cages' can be released from 
mRNA transcripts sequentially upon irradiation with $365 \mathrm{~nm} / 405 \mathrm{~nm}$ light (lamp excitation) or $405 \mathrm{~nm} / 488 \mathrm{~nm}$ light (laser excitation), leading to translational activation of the corresponding mRNA. Irradiation with a longer wavelength of light only cleaves one 'photo-cage' (biotinDEACM-preQ 1 ) from RNA, while a shorter wavelength of light cleaves both 'photo-cages'. By using the appropriate order of photo-irradiation, sequential photo-activation of two mRNAs within the same cell was demonstrated by live cell fluorescence imaging. We believe that the ability to sequentially photo-activate two genes with high spatial-temporal resolution provides a powerful and versatile optogenetic tool to build robust, complex, and scalable synthetic gene networks. Such a tool may improve capabilities to precisely manipulate biological networks, which can aid studies of gene regulatory mechanisms, promote the engineering of artificial biological systems, and facilitate the development of novel therapeutic applications.

\section{AUTHOR INFORMATION}

\section{Corresponding Author}

Neal K. Devaraj

E-mail: ndevaraj@ucsd.edu

\section{Author Contributions}

†These authors contributed equally.

\section{Notes}

The authors declare no competing financial interest.

\section{ACKNOWLEDGMENTS}

The project or effort depicted is sponsored by the Defense Advanced Research Projects Agency Biological Technologies Office (BTO) Safe Genes Program under Contract Number HR0011-182-0039. The content of the information does not necessarily reflect the position or the policy of the government, and no official endorsement should be inferred.

\section{KEYWORDS}

gene regulation, optogenetics, translation, RNA modification, multiplexing 


\section{REFERENCES}

[1] Lanctôt, C., Cheutin, T., Cremer, M., Cavalli, G., \& Cremer, T. (2007). Dynamic genome architecture in the nuclear space: regulation of gene expression in three dimensions. Nature Reviews Genetics, 8(2), 104-115.

[2] Jaenisch, R., \& Bird, A. (2003). Epigenetic regulation of gene expression: how the genome integrates intrinsic and environmental signals. Nature genetics, 33(3), 245-254.

[3] Kistner, A., Gossen, M., Zimmermann, F., Jerecic, J., Ullmer, C., Lübbert, H., \& Bujard, H. (1996). Doxycycline-mediated quantitative and tissue-specific control of gene expression in transgenic mice. Proceedings of the National Academy of Sciences, 93(20), 10933-10938.

[4] Motta-Mena, L. B., Reade, A., Mallory, M. J., Glantz, S., Weiner, O. D., Lynch, K. W., \& Gardner, K. H. (2014). An optogenetic gene expression system with rapid activation and deactivation kinetics. Nature chemical biology, 10(3), 196.

[5] Fenno, L., Yizhar, O., \& Deisseroth, K. (2011). The development and application of optogenetics. Annual review of neuroscience, 34.

[6] Deiters, A. (2009). Light activation as a method of regulating and studying gene expression. Current opinion in chemical biology, 13(5-6), 678-686.

[7] Gardner, L., \& Deiters, A. (2012). Light-controlled synthetic gene circuits. Current opinion in chemical biology, 16(3-4), 292-299.

[8] Adams, S. R., \& Tsien, R. Y. (1993). Controlling cell chemistry with caged compounds. Annual review of physiology, 55(1), 755-784.

[9] Mayer, G., \& Heckel, A. (2006). Biologically active molecules with a "light switch". Angewandte Chemie International Edition, 45(30), 4900-4921.

[10] Tang, X., \& Dmochowski, I. J. (2007). Regulating gene expression with light-activated oligonucleotides. Molecular BioSystems, 3(2), 100-110.

[11] Courtney, T., \& Deiters, A. (2018). Recent advances in the optical control of protein function through genetic code expansion. Current opinion in chemical biology, 46, 99-107.

[12] Liu, Q., \& Tucker, C. L. (2017). Engineering genetically-encoded tools for optogenetic control of protein activity. Current opinion in chemical biology, 40, 17-23.

[13] Kaberniuk, A. A., Shemetov, A. A., \& Verkhusha, V. V. (2016). A bacterial phytochromebased optogenetic system controllable with near-infrared light. Nature methods, 13(7), 591.

[14] Zetsche, B., Volz, S. E., \& Zhang, F. (2015). A split-Cas9 architecture for inducible genome editing and transcription modulation. Nature biotechnology, 33(2), 139-142.

[15] Taslimi, A., Zoltowski, B., Miranda, J. G., Pathak, G. P., Hughes, R. M., \& Tucker, C. L. (2016). Optimized second-generation CRY2-CIB dimerizers and photoactivatable Cre recombinase. Nature chemical biology, 12(6), 425.

[16] Nihongaki, Y., Kawano, F., Nakajima, T., \& Sato, M. (2015). Photoactivatable CRISPR-Cas9 for optogenetic genome editing. Nature biotechnology, 33(7), 755-760.

[17] Mikat, V., \& Heckel, A. (2007). Light-dependent RNA interference with nucleobase-caged siRNAs. Rna, 13(12), 2341-2347.

[18] Tang, X., Maegawa, S., Weinberg, E. S., \& Dmochowski, I. J. (2007). Regulating gene expression in zebrafish embryos using light-activated, negatively charged peptide nucleic acids. Journal of the American Chemical Society, 129(36), 11000-11001.

[19] Blidner, R. A., Svoboda, K. R., Hammer, R. P., \& Monroe, W. T. (2008). Photoinduced RNA interference using DMNPE-caged 2'-deoxy-2'-fluoro substituted nucleic acids in vitro and in vivo. Molecular BioSystems, 4(5), 431-440. 
[20] Tang, X., Swaminathan, J., Gewirtz, A. M., \& Dmochowski, I. J. (2008). Regulating gene expression in human leukemia cells using light-activated oligodeoxynucleotides. Nucleic acids research, 36(2), 559-569.

[21] Young, D. D., Lusic, H., Lively, M. O., Yoder, J. A., \& Deiters, A. (2008). Gene silencing in mammalian cells with light-activated antisense agents. ChemBioChem, 9(18), 2937-2940.

[22] Ouyang, X., Shestopalov, I. A., Sinha, S., Zheng, G., Pitt, C. L., Li, W. H., ... \& Chen, J. K. (2009). Versatile synthesis and rational design of caged morpholinos. Journal of the American Chemical Society, 131(37), 13255-13269.

[23] Jain, P. K., Shah, S., \& Friedman, S. H. (2011). Patterning of gene expression using new photolabile groups applied to light activated RNAi. Journal of the American Chemical Society, 133(3), 440-446.

[24] Young, D. D., Lively, M. O., \& Deiters, A. (2010). Activation and deactivation of DNAzyme and antisense function with light for the photochemical regulation of gene expression in mammalian cells. Journal of the American Chemical Society, 132(17), 6183-6193.

[25] Ogasawara, S. (2014). Control of cellular function by reversible photoregulation of translation. ChemBioChem, 15(18), 2652-2655.

[26] Shestopalov, I. A., Sinha, S., \& Chen, J. K. (2007). Light-controlled gene silencing in zebrafish embryos. Nature chemical biology, 3(10), 650-651.

[27] Wang, Y., Wu, L., Wang, P., Lv, C., Yang, Z., \& Tang, X. (2012). Manipulation of gene expression in zebrafish using caged circular morpholino oligomers. Nucleic acids research, 40(21), $11155-11162$.

[28] Govan, J. M., Young, D. D., Lusic, H., Liu, Q., Lively, M. O., \& Deiters, A. (2013). Optochemical control of RNA interference in mammalian cells. Nucleic acids research, 41(22), 10518-10528.

[29] Ando, H., Furuta, T., Tsien, R. Y., \& Okamoto, H. (2001). Photo-mediated gene activation using caged RNA/DNA in zebrafish embryos. Nature genetics, 28(4), 317-325.

[30] Sonenberg, N., \& Hinnebusch, A. G. (2009). Regulation of translation initiation in eukaryotes: mechanisms and biological targets. Cell, 136(4), 731-745.

[31] Sahin, U., Karikó, K., \& Türeci, Ö. (2014). mRNA-based therapeutics-developing a new class of drugs. Nature reviews Drug discovery, 13(10), 759.

[32] Zhang, D., Zhou, C. Y., Busby, K. N., Alexander, S. C., \& Devaraj, N. K. (2018). Lightactivated control of translation by enzymatic covalent mRNA Labeling. Angewandte Chemie International Edition, 57(11), 2822-2826.

[33] Alexander, S. C., Busby, K. N., Cole, C. M., Zhou, C. Y., \& Devaraj, N. K. (2015). SiteSpecific covalent labeling of RNA by enzymatic transglycosylation. Journal of the American Chemical Society, 137(40), 12756-12759.

[34] Ehret, F., Zhou, C. Y., Alexander, S. C., Zhang, D., \& Devaraj, N. K. (2017). Site-specific covalent conjugation of modified mRNA by tRNA guanine transglycosylase. Molecular pharmaceutics, 15(3), 737-742.

[35] Chen, Y. C., Brooks, A. F., Goodenough-Lashua, D. M., Kittendorf, J. D., Showalter, H. D., \& Garcia, G. A. (2011). Evolution of eukaryal tRNA-guanine transglycosylase: insight gained from the heterocyclic substrate recognition by the wild-type and mutant human and Escherichia coli tRNA-guanine transglycosylases. Nucleic acids research, 39(7), 2834-2844.

[36] Stengl, B., Reuter, K., \& Klebe, G. (2005). Mechanism and Substrate Specificity of tRNAGuanine Transglycosylases (TGTs): tRNA-Modifying Enzymes from the Three Different Kingdoms of Life Share a Common Catalytic Mechanism. ChemBioChem, 6(11), 1926-1939. 
[37] Hurt, J. K., Olgen, S., \& Garcia, G. A. (2007). Site-specific modification of Shigella flexneri virF mRNA by tRNA-guanine transglycosylase in vitro. Nucleic acids research, 35(14), 49054913.

[38] Curnow, A. W., Kung, F. L., Koch, K. A., \& Garcia, G. A. (1993). tRNA-guanine transglycosylase from Escherichia coli: gross tRNA structural requirements for recognition. Biochemistry, 32(19), 5239-5246.

[39] Goguen, B. N., Aemissegger, A., \& Imperiali, B. (2011). Sequential activation and deactivation of protein function using spectrally differentiated caged phosphoamino acids. Journal of the American Chemical Society, 133(29), 11038-11041.

[40] Yamazoe, S., Liu, Q., McQuade, L. E., Deiters, A., \& Chen, J. K. (2014). Sequential gene silencing using wavelength-selective caged morpholino oligonucleotides. Angewandte Chemie International Edition, 53(38), 10114-10118.

[41] Holstein, J. M., Anhäuser, L., \& Rentmeister, A. (2016). Modifying the 5'-cap for click reactions of eukaryotic mRNA and to tune translation efficiency in living cells. Angewandte Chemie International Edition, 55(36), 10899-10903. 